\title{
Evaluation of the role of LRRK2 gene in Parkinson's disease in an East Indian cohort
}

\author{
Tamal Sadhukhan $^{\mathrm{a}}$, Mansi Vishal ${ }^{\mathrm{b}}$, Gautami Das ${ }^{\mathrm{a}}$, Aanchal Sharma ${ }^{\mathrm{c}}$, Arijit Mukhopadhyay ${ }^{\mathrm{c}}$, \\ Shyamal K. Das ${ }^{\mathrm{d}}$, Kunal Ray ${ }^{\mathrm{b}, *}$ and Jharna Ray ${ }^{\mathrm{a}, *}$ \\ ${ }^{a}$ S. N. Pradhan Centre for Neurosciences, University of Calcutta, Kolkata, India \\ ${ }^{\mathrm{b}}$ Molecular and Human Genetics Division, CSIR-Indian Institute of Chemical Biology, Kolkata, India \\ ${ }^{\mathrm{c}}$ Genomics and Molecular Medicine, CSIR-Institute of Genomics and Integrative Biology, Delhi \\ ${ }^{\mathrm{d}}$ Movement Disorders Clinic, Bangur Institute of Neurosciences, Kolkata, India
}

\begin{abstract}
Leucine rich repeat kinase 2 (LRRK2) gene defects cause Parkinson's disease (PD). Recently, LRRK2 has also been shown by genome wide association (GWA) studies to be a susceptibility gene for the disease. In India mutations in $L R R K 2$ is a rare cause of PD. We, therefore, genotyped 64 SNPs across $L R R K 2$ in 161 control samples and finally studied 6 haplotype tagging SNPs for association-based study on 300 cases and 446 ethnically matched controls to explore the potential role of $L R R K 2$ as a susceptibility gene in PD for East Indians. We did not find any significant allele/ genotype or haplotype associations with PD suggesting that common genetic variants within LRRK2 play limited role in modulating PD among East Indians. In addition, we also screened for the common mutations (viz. p.R1441C, p.R1441G, p.R1441H, p.Y1699C, p.G2019S), and a risk variant common among Asians (p.G2385R) but did not observe any of the above mentioned variants in our cohort. Our study, therefore, strongly suggests that $L R R K 2$ has minimal role as a candidate and susceptibility gene in PD pathogenesis among East Indians.
\end{abstract}

Keywords: Haplotype, LRRK2, mutation, Parkinson's disease, SNP

\section{Introduction}

Parkinson's disease (PD) [MIM \#168600] is an age related neurodegenerative disease with complex etiology affecting $2 \%$ of the global population over the age of 65 [2]. Mutations in the LRRK2 gene [MIM \#609007] encoding leucine-rich repeat kinase 2 have been reported to be linked with autosomal dominant form of familial PD and these mutants clinically resemble idiopathic PD [6]. Most of the pathogenic mutations of $L R R K 2$ are located in the different functional domains of the protein and they account for $\sim 6 \%$ of the autosomal dominant form of PD [12]. But because of its large size (51 exons spanning $145 \mathrm{~kb}$ region on

*Corresponding authors: Dr. Kunal Ray, Molecular \& Human Genetics Division, CSIR-Indian Institute of Chemical Biology, 4 Raja S. C. Mullick Road, Kolkata - 700 032, India. Tel.: +91 33 2483 1984; Fax: +91 332473 5197; E-mail: kunalray@gmail.com, kray@iicb.res.in; Prof. Jharna Ray, S. N. Pradhan Centre for Neurosciences, University of Calcutta, 35, Ballygunge Circular Road, Kolkata - 700 019, India. Tel.: +91 332223 2084; Fax: +91 332223 3260; E-mail: jharnaray@gmail.com, thisisjr@ rediffmail.com. chromosome 12p11.2-q13.1), the gene screening for the mutations remained restricted largely to common LRRK2 defects implicated in PD pathogenesis. This approach does not, however, rule out the presence of other mutations or risk variants that might be associated with the disease pathogenesis. Using tagSNPs, a risk haplotype has been identified for sporadic PD in a Chinese cohort [18]. Subsequently, a common risk variant (p.G2385R) was found to be associated among the Asians (Chinese, Japanese, and Taiwanese populations) but not in Caucasians and this was supported by a recent study involving 1376 PD and 962 controls from Asia [14]. Recent genome wide association (GWA) studies indicate that like the candidate gene alpha synuclein, LRRK2 is also a susceptibility locus for PD suggesting the importance of studying this gene for its potential to qualify as a susceptibility gene in PD in different population groups [16,17].

In India there have been 3 studies on LRRK2 from different geographical regions (north, east and south); however these studies have only searched for the most prevalent (p.G2019S) or common mutations and have 
Table 1

Demographic features of PD patients and controls

\begin{tabular}{lcc}
\hline & Patient & Control \\
\hline Total study subjects $(n)$ & 300 & 446 \\
Mean age* $(\mathrm{Yr} \pm \mathrm{SD})$ & $53.71 \pm 12.07$ & $49.58 \pm 10.78$ \\
Mean age of onset $(\mathrm{Yr} \pm \mathrm{SD})$ & $48.48 \pm 12.17$ & - \\
Male/Female & $234 / 66$ & $288 / 158$ \\
Familial/Sporadic & $88 / 182^{\S}$ & - \\
Older Age Group & LOPD $(>40)$ & Control $(>45)$ \\
Study subjects & 214 & 273 \\
Mean age of onset $(\mathrm{Yr} \pm \mathrm{SD})$ & $54.44 \pm 8.36$ & - \\
Younger Age Group & YOPD $(\leqslant 40)$ & Control $(\leqslant 45)$ \\
Study subjects & 86 & 173 \\
Mean age of onset $(\mathrm{Yr} \pm \mathrm{SD})$ & $33.62 \pm 5.78$ & - \\
\hline
\end{tabular}

*Age at the time of examination; $\S$, Family history of rest of 30 cases was not known, LOPD, Late onset PD; YOPD, Young onset PD.

not explored the role of $L R R K 2$ as a susceptibility gene in PD $[13,15,21]$. Herein, we report on a comprehensive, haplotype-tagging SNP approach to determine whether LRRK2 is a susceptibility gene for PD in an East Indian cohort. In addition, we screened the gene for the presence of the common mutations and a risk variant of $L R R K 2$ in this cohort.

\section{Materials and methods}

\subsection{Study subjects}

In this study 300 clinically diagnosed PD patients with a mean age at onset of $48.48 \pm 12.17$ years (age range 20-77 years) from East India and 446 ethnically and age matched controls (mean age, $49.58 \pm$ 10.78 years), having no personal or family history of any neurodegenerative diseases were recruited. Demographic features of all study subjects are shown in Table 1. All patients underwent detailed neurological examination at the Movement disorders Clinic at Bangur Institute of Neurosciences, Kolkata, India, and patients having at least 2 cardinal symptoms (rest tremor, bradykinesia, rigidity and postural instability) were selected for the study. The study protocol adhered to the tenets of the Declaration of Helsinki and the institutional review board on research using human subject approved the study after proper review as per regulation of the Indian Council of Medical Research.

\subsection{Genomic DNA isolation and genotyping of prevalent mutations}

Genomic DNA was prepared from peripheral blood by conventional salting-out method using sodium per- chlorate followed by isopropanol precipitation [7]. $L R$ $R K 2$ exons $31,35,41$ and 48 and their adjacent flanking sequences were amplified by PCR in a total volume of $25.0 \mu \mathrm{l}$ containing 40-100 ng genomic DNA, $0.4 \mu \mathrm{M}$ of each primer, $0.2 \mathrm{mM}$ of each dNTP, $1.0-1.5 \mathrm{mM}$ of $\mathrm{MgCl}_{2}$ (as appropriate) and 0.5 unit of Taq polymerase (Invitrogen, Carlsbad, California) in a Thermocycler (GeneAmp-2700, PE Applied Biosystems, Foster City, CA, USA). The PCR amplicons were analyzed on $6 \%$ polyacrylamide gel and visualized by ethidium bromide staining. For quick screening of nucleotide variants, PCR products were subjected to Single Stranded Conformation Polymorphism (SSCP) analysis. Samples showing band shifts were subjected to bi-directional DNA sequencing to identify the nucleotide variant. The presence of mutation p.G2019S was examined in entire patient cohort by RFLP analysis [13].

\subsection{Selection of tagSNP and genotyping}

Since PCA (Principal Component Analysis) plots suggest that Indians are much more closely related to the Caucasians rather than the Asians, we therefore selected tagSNPs of LRRK2 that are specific for the HapMap (hapmap.ncbi.nlm.nih.gov) CEU population in the present study. Out of the 20 tagSNPs in the CEU population, 5 were selected viz. rs6581622, rs2723264, rs7957057, rs12367542 and rs10878405 (p.E2108E) for association based study in 256 cases and 446 controls. The selection was based upon the criteria that the SNPs have lower LD value $\left(r^{2}<0.8\right)$ between them, greater coverage across the gene, uniformly spaced, and have high heterozygosity.

The selected tagSNPs were genotyped by polymerase chain reaction $(\mathrm{PCR})$ - restriction fragment length polymorphism (RFLP) method (primer sequences and details of RFLP are available upon request). In case, where there was no restriction site, one of the 2 primers had a mismatch that artificially created a restriction site. About $20 \%$ of the samples were randomly selected and their genotypes were reconfirmed by DNA sequencing.

Later, we had the opportunity to get the genotyped data of 59 additional SNPs in 161 individuals of our control subjects that were part of an independent project involving high-throughput genotyping on Human660wQuad-v1 chip (Illumina). This chip contained all tag SNPs designed by taking HapMap population as a reference. About 200 ng DNA was loaded on the chip for genotyping. Chip scanning was done by Illumina Beadxpress reader while genotype calling was 
performed by Genotyping module of Genome studio software provided by Illumina. Initial quality checks involved $98 \%$ call rate for samples and $95 \%$ for SNPs. In addition samples were checked for relatedness, followed by 0.01 of minor allele frequency (MAF) and checked for Hardy-Weinberg Equilibrium $(p<0.01)$. From the LD pattern generated from the frequencies of all the 64 studied SNPs across LRRK2 we selected another SNP rs3761863 (p.M2397T) for association study.

\subsection{Statistical analysis}

Haploview 4.1 was used to test the LD block and predict the haplotypes for the SNPs [1]. For association study the data were evaluated for p-value, odds ratio (OR), and $95 \%$ confidence interval (CI) by using the "Online Statistical Calculations" (statpages. org/ctab2x2.html).

\section{Results}

\subsection{Screening of prevalent mutations and p.G2358R polymorphism}

Our study on screening for the common pathogenic mutations (viz. p.R1441C, p.R1441G, p.R1441H, p.Y1699C, p.G2019S) in LRRK2 gene among 300 patient samples failed to identify any of these variants. In addition two other mutations (p.I2012T and p.I2020T) reported in Taiwanese and Japanese populations [19] and located within the coding sequence scanned for p.G2019S were not found. However, we detected a few intronic variants viz. IVS34-51A $>\mathrm{T}$ (rs10878368), IVS35+23T $>$ A (rs7307276), IVS479 Tdel (rs11317573), IVS48+52G $>$ A. Interestingly, the functional polymorphism, p.G2358R that has been found to be associated with PD exclusively in Asian populations was found to be monomorphic in our case and control cohorts.

\subsection{LD pattern of selected SNPs in LRRK2 gene}

The pairwise LD pattern of all the 64 selected SNPs across LRRK2 gene was calculated (Fig. 1). The 6 SNP markers were chosen for association study; of which 4 (rs6581622, rs2723264, rs12367542 and rs3761863) were found to be tagSNPs in our population suggesting that these SNPs would represent a larger genomic region of $L R R K 2$.

\subsection{Association study}

All the 6 SNPs were within Hardy-Weinberg equilibrium in both cases and controls. We next evaluated the genetic association of these SNPs in 256 PD patients and 437 controls (Table 2). However, we could not find any significant association $(p>0.05)$ with the disease. LRRK2 haplotypes were then constructed using the 6 SNPs but no significant variation in the haplotype frequency between cases and controls was observed (Table 3). Since increasing age and positive family history are the 2 major risk factors for PD, we categorized the patients into early onset (age of onset $\leqslant 40$ years), late onset (age of onset $>40$ years), those with a family history of any kind of neurological problems (e.g. PD, dystonia, tremor, etc.), and sporadic cases. The controls were similarly grouped as young (age $\leqslant 45$ years) and aged (age $>45$ years). Here also, on comparing the different subgroups of patients and controls we did not find significant association of PD with any specific allele, genotype or haplotype.

\section{Discussion}

In the present study we have tried to dissect the role of LRRK2 as a candidate and susceptibility gene in PD pathogenesis among eastern Indians. The most common genetic mutation in PD (p.G2019S of LRRK2) accounts for $6.6 \%$ of familial and $1.6 \%$ of sporadic PD cases in Caucasians [3,5]. Its frequency is high in the North African Arabs (40\%) [9], and Ashkenazi Jews (30\%) [11], moderate in the Portuguese (8-9\%) and very low $(<1 \%)$ in countries like Austria, Belgium, Denmark, Germany, Greece, Japan, India, Iran and Poland [10]. Moreover, p.G2019S has an age dependent penetrance, increasing from $17 \%$ at age of 50 years to $85 \%$ at age of 70 years [8]. Our study demonstrates that the common mutations of LRRK2 (p.R1441C, p.R1441G, p.R1441H, p.Y1699C, p.G2019S) are absent in this cohort suggesting that these mutations in $L R R K 2$ among PD cases is population specific.

Studies on LRRK2 have been done on a few selected variants in the Indian population encompassing northern [13], southern [21] and eastern regions [15] of the country. Among these variants p.Gly2019Ser has been extensively studied in all the 3 regions of India (including the present one). Till date 1444 PD patients have been screened across India (including the present study) for this mutation in $L R R K 2$, however, only one female patient from northern India has been reported to 


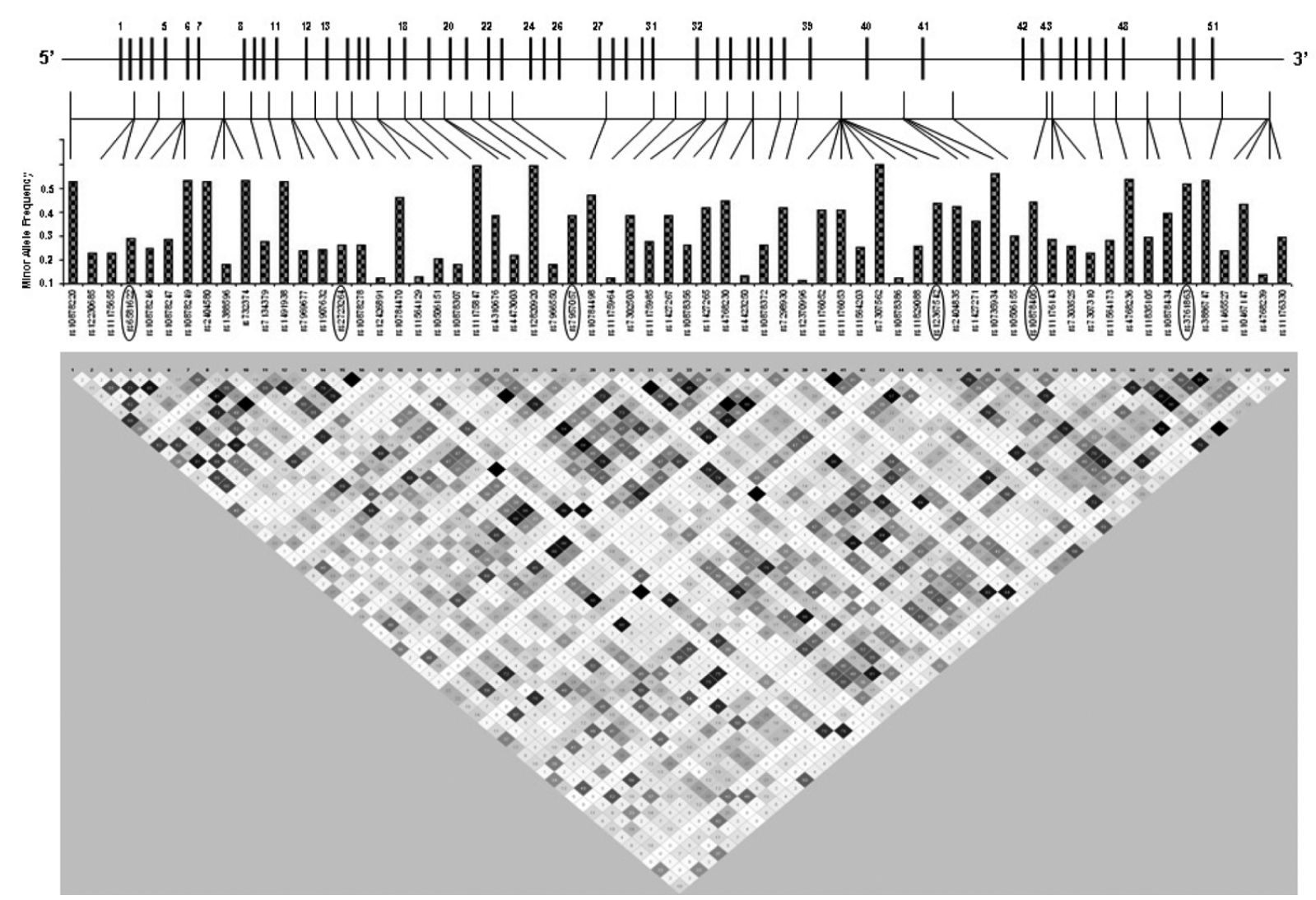

Fig. 1. Minor allele frequencies (MAF) and LD plot of all 64 SNPs across LRRK2 gene. Those SNPs selected for association study are encircled. The vertical thick lines represent exons (1-51 in LRRK2 gene). Exons and introns are not drawn to scale.

harbor the mutation in heterozygous state [13]. Other common mutations like p.Arg1441Cys, p.Arg1441Gly, p.Arg1441His, p.Tyr1699Cys have been studied in the North and East Indian populations (including the present study). Among East Indians we and Sanyal et al. [15] have examined for an additional mutation, p.Tyr1699Cys but did not find one. Our result on common mutation screening in LRRK2 is consistent with 3 previous studies suggesting that at least previously reported mutations in other populations must be rare, if not absent, among Indians. However, despite the size of the gene and a large number of exons, screening for mutation in the entire gene is warranted to truly determine the role of the gene in pathogenesis of PD among Indians. One would expect that with the advent of high throughput sequencing with gradual slump of cost involved, such studies would be practical with relatively modest budget in near future.

The functional polymorphism, p.G2385R (c.7153G $>$ A) has been found to be a potential risk factor for PD among Asians (Japanese/ Chinese ancestry) but rarely found among Caucasians [4,14]. Another study was done among 472 Asian subjects from Malay and Indian ethnicity (non Chinese /Japanese population), where only 2 heterozygous carriers of p.G2385R were found in each among 98 Malay PD and 173 Malay controls, but not in 66 Indian PD patients and 133 controls [20]. The residue G2385 is located in the WD40 domain of LRRK2, which is involved in a variety of cellular functions such as signal transduction, pre-mRNA processing and cytoskeleton assembly. The $\mathrm{G} 2385$ residue is exposed at the surface of WD40 domain, which could either prevent protein interaction or lead to generation of new interaction with other proteins. In our study we did not find this Asian specific p.G2385R risk variant among 200 PD cases of eastern India. This is also the first study on p.G2385R variant from India and emphasizes the fact that it is monomorphic in this population and therefore less likely to provide different results in future studies.

The common mutations of LRRK2 being absent in our population does not rule out the possibility of $L R$ $R K 2$ being a susceptibility gene in PD. Hence, apart from studying the risk variant p.G2385R, we took 


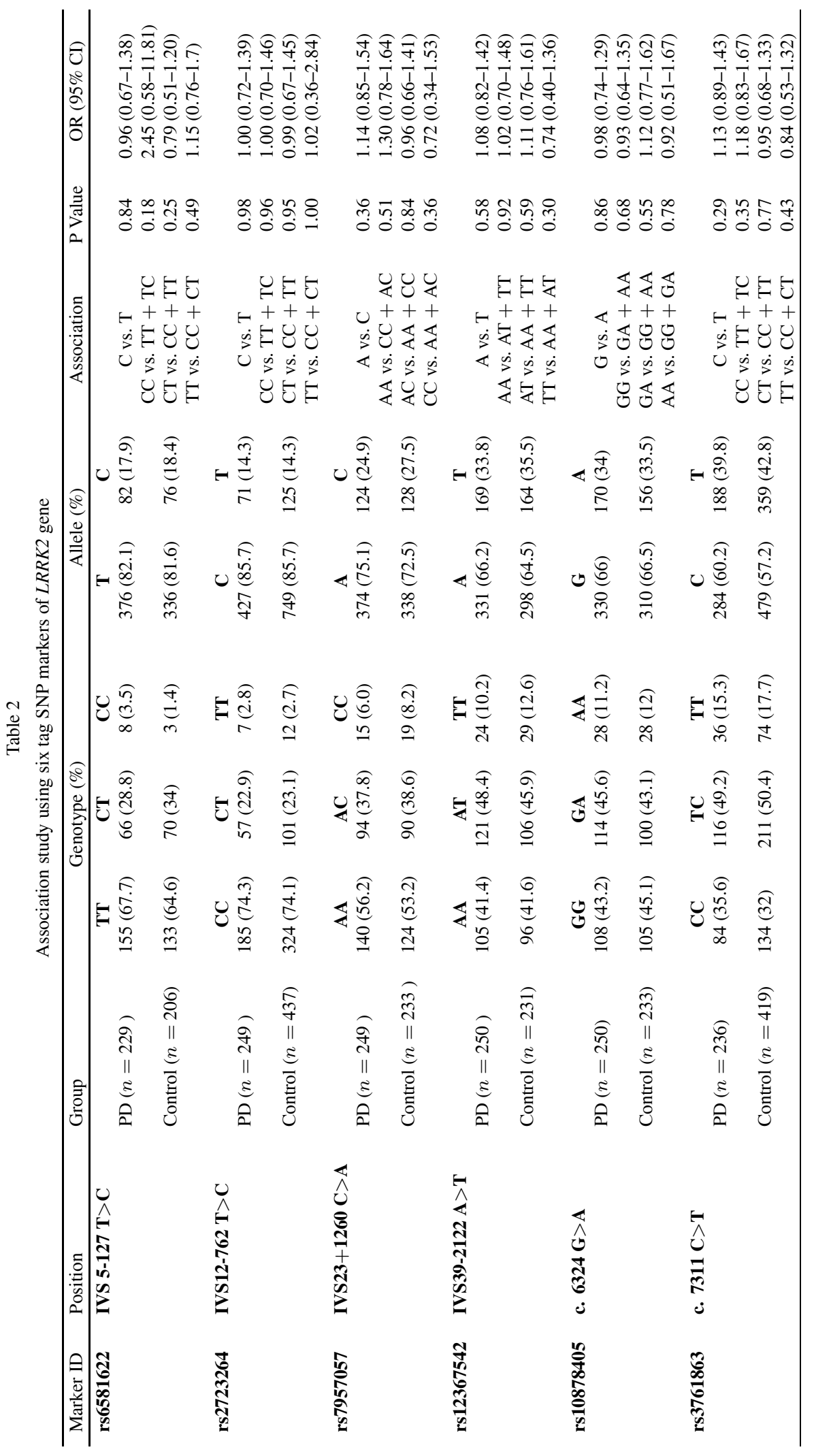




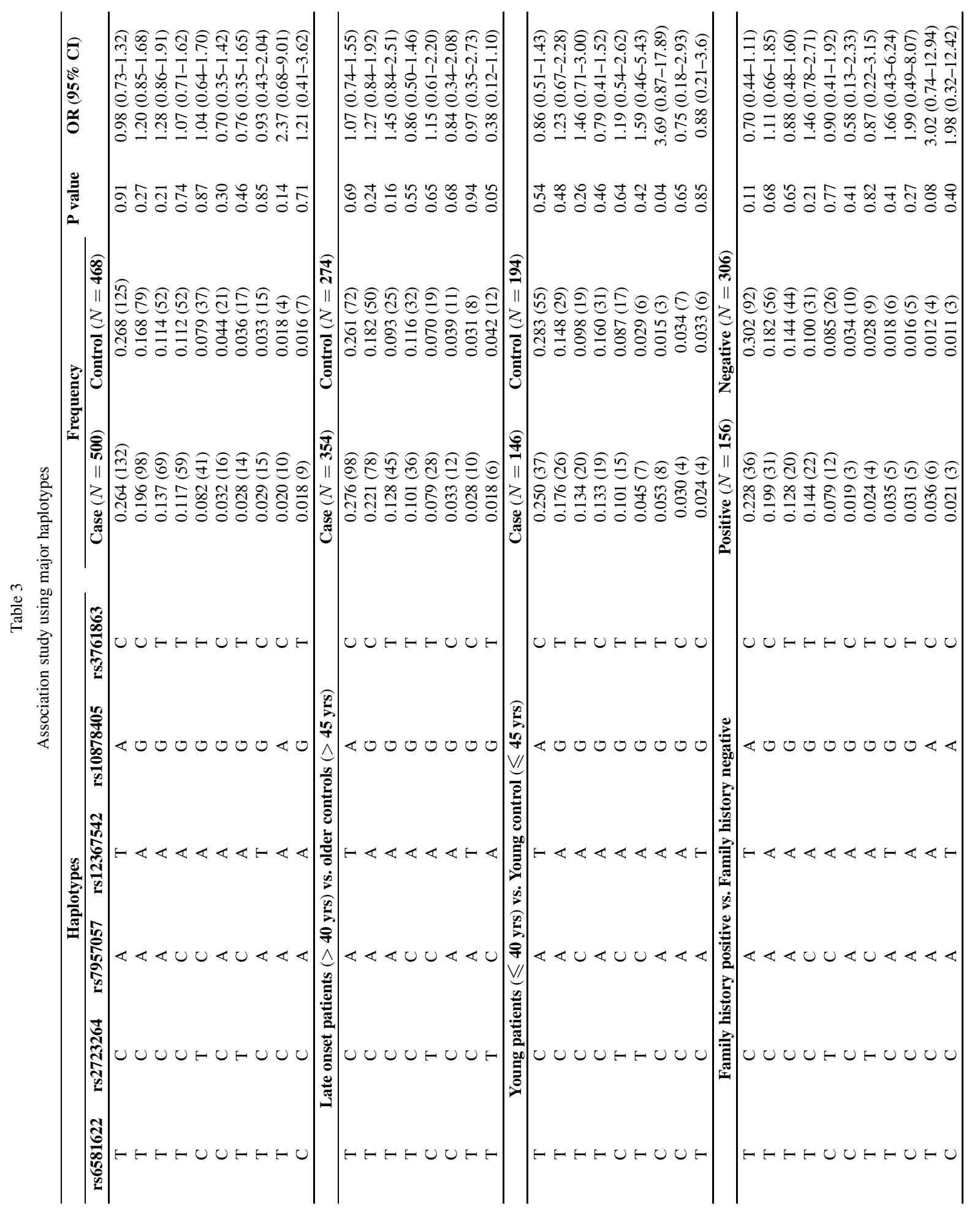


haplotype-tagging SNP (htSNP) approach to evaluate the entire locus with the fewest number of maximally informative SNPs so as to understand the role of $L R R K 2$ in PD pathogenesis in this cohort. Unlike the recent GWA studies $[16,17]$ we did not find any contribution of $L R R K 2$ as a susceptibility gene among the Indians. The SNPs found to be associated in GWA studies [16, 17], located 18 to $180 \mathrm{~kb}$ upstream of $L R R K 2$, were examined by Simon-Sanchez et al. for expression of the gene but was not reported to have any effect [17]. To explore the possibility that the SNPs are in LD with down stream variants in LRRK2 gene, which might have effect on the biological activity of the gene product, we analyzed the genotype data for the SNPs in our control samples but did not observe any significant LD. Therefore, it is unlikely that the SNPs upstream to $L R$ $R K 2$ reported by GWA studies [16,17] are associated in our PD cohort. Although no significant association (allelic/ genotypic/ haplotype) was identifiable in this study, our study is likely to represent the true picture of the population specific variability within LRRK2 gene among East Indians. Since different sets of SNPs are associated with PD risk in diverse ethnic groups, our selection of 3 out of the 5 tagSNPs from the CEU population were later found to be tagSNPs in the Eastern Indian population as well. This actually provides a greater confidence in the variability within the gene in this population. However, our study does not exclude the possibility of less common genetic variables within LRRK2 in influencing PD.

In conclusion, this comprehensive htSNP study found no evidence of $L R R K 2$ in influencing PD risk nor could we identify any of the prevalent mutations and a functional risk polymorphism in this cohort implying that $L R R K 2$ plays limited role in PD pathogenesis among Indians.

\section{Acknowledgements}

The authors are thankful to all patients and healthy volunteers for participating in this study. The study was supported partially by grants from University Grant Commission [grant No. F.14-38/2007(Inno./ASIST)] and Council of Scientific and Industrial Research (CSIR) (grant no. MLP-0016 and SIP-007). TS is supported by a pre-doctoral fellowship from the Government of West Bengal, India.

\section{References}

[1] J.C. Barrett, B. Fry, J. Maller and M.J. Daly, Haploview: analysis and visualization of LD and haplotype maps, Bioinformatics 21 (2005), 263-265.

[2] M.C. de Rijk, L.J. Launer, K. Berger, M.M. Breteler, J.F. Dartigues, M. Baldereschi, L. Fratiglioni, A. Lobo, J. MartinezLage, C. Trenkwalder and A. Hofman, Prevalence of Parkinson's disease in Europe: A collaborative study of populationbased cohorts. Neurologic Diseases in the Elderly Research Group, Neurology 54 (11 Suppl 5) (2000), 21-23.

[3] A. Di Fonzo, C.F. Rohé, J. Ferreira, H.F. Chien, L. Vacca, F. Stocchi, L. Guedes, E. Fabrizio, M. Manfredi, N. Vanacore, S. Goldwurm, G. Breedveld, C. Sampaio, G. Meco, E. Barbosa, B.A. Oostra, V. Bonifati and Italian Parkinson Genetics Network, A frequent LRRK2 gene mutation associated with autosomal dominant Parkinson's disease, Lancet 365 (2005), 412-415.

[4] M. Funayama, Y. Li, H. Tomiyama, H. Yoshino, Y. Imamichi, M. Yamamoto, M. Murata, T. Toda, Y. Mizuno and N. Hattori, Leucine-rich repeat kinase $2 \mathrm{G} 2385 \mathrm{R}$ variant is a risk factor for Parkinson disease in Asian population, Neuroreport 18 (2007), 273-275.

[5] W.P. Gilks, P.M. Abou-Sleiman, S. Gandhi, S. Jain, A. Singleton, A.J. Lees, K. Shaw, K.P. Bhatia, V. Bonifati, N.P. Quinn, J. Lynch, D.G. Healy, J.L. Holton, T. Revesz and N.W. Wood, A common LRRK2 mutation in idiopathic Parkinson's disease, Lancet 365 (2005), 415-416.

[6] K. Haugarvoll and Z.K. Wszolek, Clinical features of LRRK2 parkinsonism, Parkinsonism Relat Disord 15 (Suppl 3) (2009), 205-208.

[7] M.B. Johns Jr. and J.E. Paulus-Thomas, Purification of human genomic DNA from whole blood using sodium perchlorate in place of phenol, Anal Biochem 180 (1989), 276-278.

[8] J. Kachergus, I.F. Mata, M. Hulihan, J.P. Taylor, S. Lincoln, J. Aasly, J.M. Gibson, O.A. Ross, T. Lynch, J. Wiley, H. Payami, J. Nutt, D.M. Maraganore, K. Czyzewski, M. Styczynska, Z.K. Wszolek, M.J. Farrer and M. Toft, Identification of a novel LRRK2 mutation linked to autosomal dominant parkinsonism: evidence of a common founder across European populations, Am J Hum Genet 76 (2005), 672-680.

[9] S. Lesage, A. Dürr, M. Tazir, E. Lohmann, A.L. Leutenegger, S. Janin, P. Pollak, A. Brice and French Parkinson's Disease Genetics Study Group, LRRK2 G2019S as a cause of Parkinson's disease in North African Arabs, N Engl J Med 354 (2006), 422-423.

[10] K. Nuytemans, J. Theuns, M. Cruts and C. Van Broeckhoven, Genetic etiology of Parkinson disease associated with mutations in the SNCA, PARK2, PINK1, PARK7, and LRRK2 genes: a mutation update, Hum Mutat 31 (2010), 763-780.

[11] L.J. Ozelius, G. Senthil, R. Saunders-Pullman, E. Ohmann, A. Deligtisch, M. Tagliati, A.L. Hunt, C. Klein, B. Henick, S.M. Hailpern, R.B. Lipton, J. Soto-Valencia, N. Risch and S.B. Bressman, LRRK2 G2019S as a cause of Parkinson's disease in Ashkenazi Jews, N Engl J Med 354 (2006), 424-425.

[12] L.M. Pardo and C.M. van Duijn, In search of genes involved in neurodegenerative disorders, Mutat Res 592 (2005), 89-101.

[13] S. Punia, M. Behari, S.T. Govindappa, P.V. Swaminath, S. Jayaram, V. Goyal, U.B. Muthane, R.C. Juyal and B.K. Thelma, Absence/rarity of commonly reported LRRK2 mutations in Indian Parkinson's disease patients, Neurosci Lett 409 (2006), 83-88.

[14] O.A. Ross, A.I. Soto-Ortolaza, M.G. Heckman et al., Association of LRRK2 exonic variants with susceptibility to Parkin- 
son's disease: a case-control study, Lancet Neurol (10) 2011, 898-908.

[15] J. Sanyal, B. Sarkar, S. Ojha, T.K. Banerjee, B.C. Ray and V.R. Rao, Absence of commonly reported leucine-rich repeat kinase 2 mutations in East Indian Parkinson's disease patients, Genet Test Mol Biomarkers 14 (2010), 691-694.

[16] W. Satake, Y. Nakabayashi, I. Mizuta, Y. Hirota, C. Ito, M. Kubo, T. Kawaguchi, T. Tsunoda, M. Watanabe, A. Takeda, H. Tomiyama, K. Nakashima, K. Hasegawa, F. Obata, T. Yoshikawa, H. Kawakami, S. Sakoda, M. Yamamoto, N. Hattori, M. Murata, Y. Nakamura and T. Toda, Genome-wide association study identifies common variants at four loci as genetic risk factors for Parkinson's disease, Nat Genet 41 (2009), 1303-1307.

[17] J. Simón-Sánchez, C. Schulte, J.M. Bras, M. Sharma, J.R. Gibbs, D. Berg, C. Paisan-Ruiz, P. Lichtner, S.W. Scholz, D.G. Hernandez, R. Krüger, M. Federoff, C. Klein, A. Goate, J. Perlmutter, M. Bonin, M.A. Nalls, T. Illig, C. Gieger, H. Houlden, M. Steffens, M.S. Okun, B.A. Racette, M.R. Cookson, K.D. Foote, H.H. Fernandez, B.J. Traynor, S. Schreiber, S. Arepalli, R. Zonozi, K. Gwinn, M. van der Brug, G. Lopez, S.J. Chanock, A. Schatzkin, Y. Park, A. Hollenbeck, J. Gao, X. Huang, N.W. Wood, D. Lorenz, G. Deuschl, H. Chen, O.
Riess, J.A. Hardy, A.B. Singleton and T. Gasser, Genome-wide association study reveals genetic risk underlying Parkinson's disease, Nat Genet 41 (2009), 1308-1312.

[18] L. Skipper, Y. Li, C. Bonnard, R. Pavanni, Y. Yih, E. Chua, W.K. Sung, L. Tan, M.C. Wong, E.K. Tan and J. Liu, Comprehensive evaluation of common genetic variation within LRRK2 reveals evidence for association with sporadic Parkinson's disease, Hum Mol Genet 14 (2005), 3549-3556.

[19] H. Tomiyama, Y. Li, M. Funayama, K. Hasegawa, H. Yoshino, S. Kubo, K. Sato, T. Hattori, C.S. Lu, R. Inzelberg, R. Djaldetti, E. Melamed, R. Amouri, N. Gouider-Khouja, F. Hentati, Y. Hatano, M. Wang, Y. Imamichi, K. Mizoguchi, H. Miyajima, F. Obata, T. Toda, M.J. Farrer, Y. Mizuno and N. Hattori, Clinicogenetic study of mutations in LRRK2 exon 41 in Parkinson's disease patients from 18 countries, Mov Disord 21 (2006), 1102-1108.

[20] E.K. Tan, Y. Zhao, L. Tan, H.Q. Lim, J. Lee, Y. Yuen, R. Pavanni, M.C. Wong, S. Fook-Chong and J.J. Liu, Analysis of LRRK2 Gly2385Arg genetic variant in non-Chinese Asians, Mov Disord 22 (2007), 1816-1818.

[21] B. Vijayan, S. Gopala and A. Kishore, LRRK2 G2019S mutation does not contribute to Parkinson's disease in South India, Neurol India 59 (2011), 157-160. 


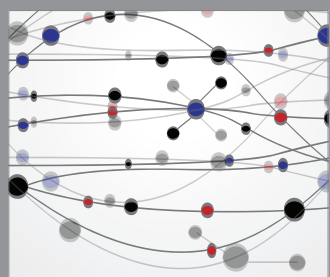

The Scientific World Journal
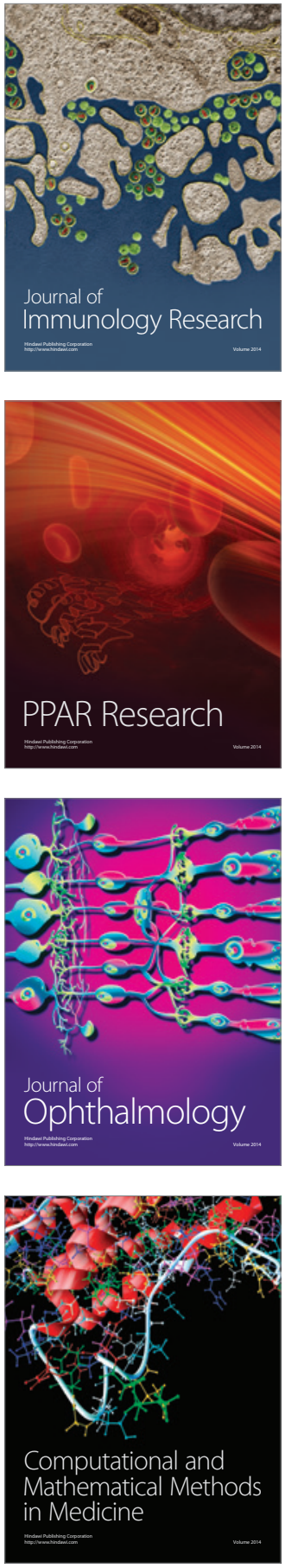

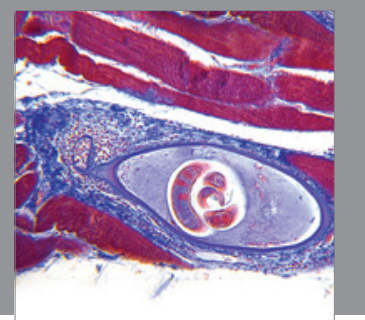

Gastroenterology

Research and Practice
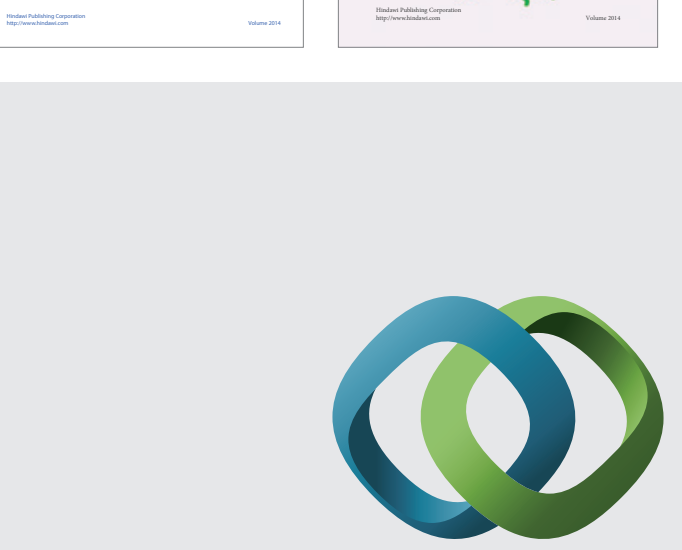

\section{Hindawi}

Submit your manuscripts at

http://www.hindawi.com
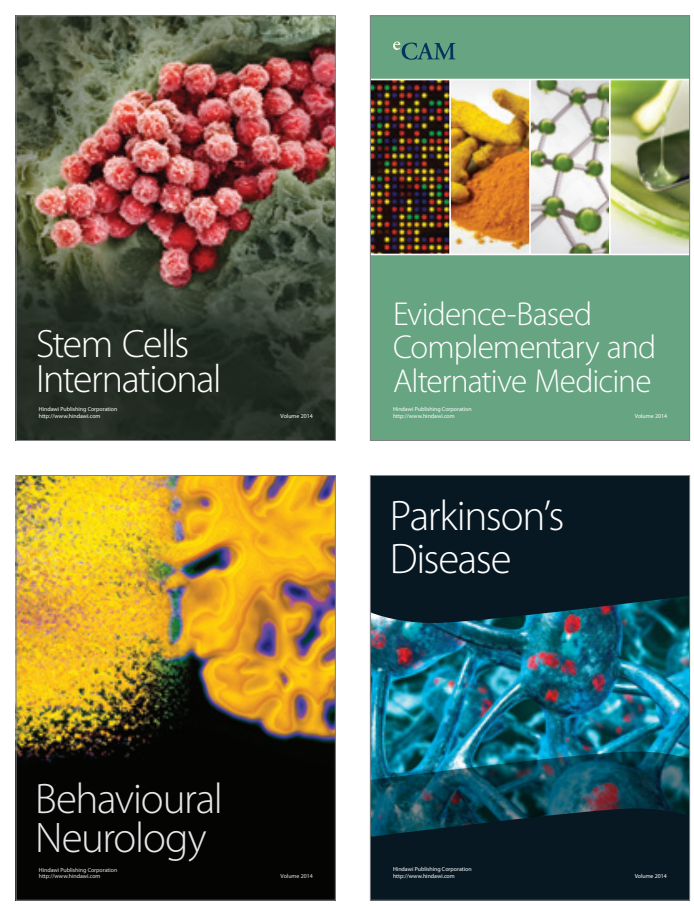

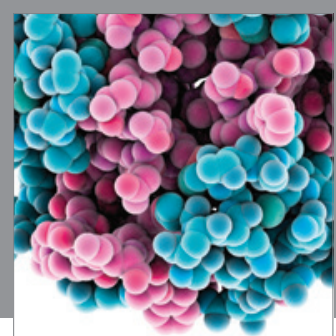

Journal of
Diabetes Research

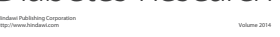

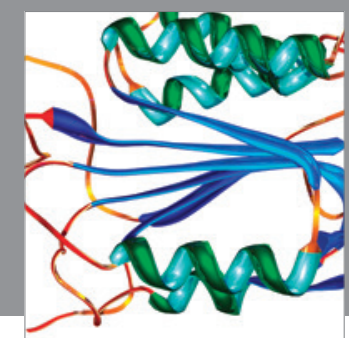

Disease Markers
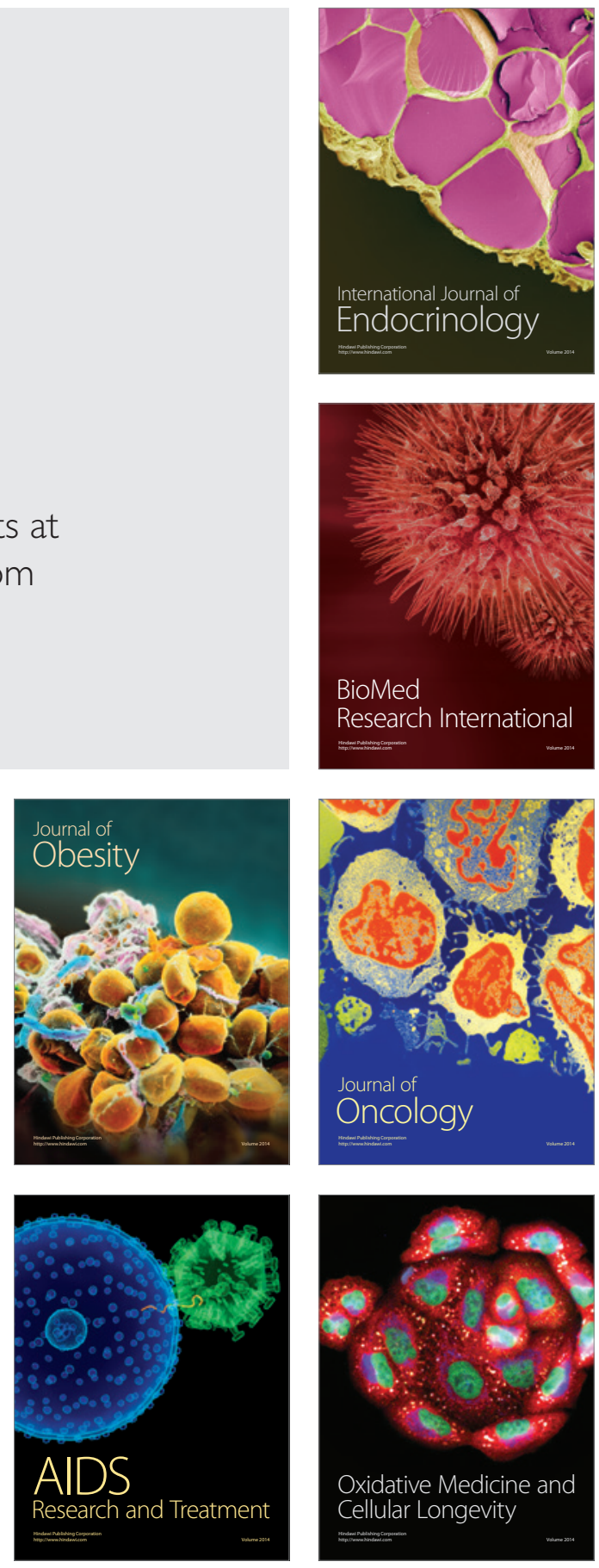